\title{
Juristische Aspekte der ärztlichen Leichenschau
}

\author{
Die Leichenschau ist der letzte ärztliche Dienst am Menschen. Prinzipiell ist jeder \\ Arzt verpflichtet, sie durchzuführen - egal ob niedergelassen oder in der Klinik. \\ Auf die leichte Schulter nehmen sollte man diese Aufgabe nicht: Wer bei der \\ Feststellung der Todesursache nicht sorgfältig vorgeht, dem drohen hohe Geld- oder \\ gar Freiheitsstrafen. Wissen Sie, welche Rechte und Pflichten Sie bei der \\ Durchführung einer ärztlichen Leichenschau haben?
}

\section{Sinn und Nutzen der Leichenschau}

Rechtssicherheit | Nur ein Arzt ist in Deutschland dazu befugt, den Tod eines Menschen festzustellen und die Leichenschau durchzuführen - und das aus gutem Grund. Schließlich fallen bei ihr Entscheidungen, die weitreichende Konsequenzen nach sich ziehen können. Die Leichenschau leistet einen Beitrag zur Rechtssicherheit, denn durch sie werden rechtsrelevante Umstände den Strafverfolgungsbehörden gemeldet und Ermittlungen überhaupt erst ermöglicht.

Erbrecht und Todesursachenstatistik | Eine sachgerecht durchgeführte Leichenschau kann weitere Todesfälle verhindern - zum Beispiel indem eine Kohlenmonoxidintoxikation erkannt und die Vergiftungsquelle beseitigt wird. Darüber hinaus lassen sich in manchen Fällen mithilfe der Leichenschau und der Obduktion Fragen des Erbrechtes oder Ansprüche Angehöriger auf Versicherungsleistungen klären. Auch stützt sich die amtliche Todesursachenstatistik auf die in den Todesbescheinigungen enthaltenen Angaben und hat Einfluss auf gesundheitspolitische Entscheidungen.

Sichere Todeszeichen | Zweifellos handelt es sich also bei der ärztlichen Leichenschau um einen äußerst verantwortungsvollen letzten Dienst am Patienten. Dazu gehört die Feststellung des Todes anhand mindestens eines sicheren Todeszeichens oder gegebenenfalls dem Hirntod. Zu den sicheren Todeszeichen zählen:

- Totenflecken

- Totenstarre

- Fäulnis

- nicht mit dem Leben vereinbare Verletzungen Außerdem werden Todesursache, Todesart und Todeszeit unter Beachtung von Meldepflichten und seuchenhygienischen Aspekten erfasst.

Gesetzliche Bestimmung der Leichenschau | Eine bundeseinheitliche gesetzliche Regelung der Leichenschau existiert nicht. Jedes Bundesland regelt die gesetzlichen Bestimmungen der Leichenschau selbst (Art. 70 Abs. 1 Grundgesetz). So ergeben sich Gemeinsamkeiten und Unterschiede bei den geltenden Gesetzen und Verordnungen.
Um die Regelungen des eigenen Bundeslandes anwenden zu können, obliegt es jedem Arzt, sich die für seine Praxis relevanten gesetzlichen Vorgaben in den Gesetzen über das Leichen-, Friedhofs- und Bestattungswesen oder in den Verordnungen des Polizei- und Ordnungsrechts anzueignen.

Im Folgenden soll auf die übereinstimmenden und grundlegenden Bestimmungen der Bundesländer eingegangen werden.

\section{Die Natur der Leiche}

Definition des Leichnams | Der Begriff des Leichnams bezieht sich auf den sich noch in geweblichem Zusammenhang befindlichen Körper einer verstorbenen Person. Knochenreste sind demnach nicht als Leichnam im Sinne des Gesetzes zu bezeichnen und bedürfen keiner Leichenschau. Darüber hinaus gilt in der Mehrzahl der Bundesländer auch ein Körperteil, ohne den ein Weiterleben ausgeschlossen ist (Rumpf oder Kopf), als Leichnam.

Tote Neugeborene I Bei toten Neugeborenen handelt es sich um verstorbene Lebendgeborene, wenn Herzschlag, Atmung oder Nabelschnurpulsation außerhalb des Mutterleibes vorhanden waren. Wenn keine Lebenszeichen vorlagen, spricht man von Totgeborenen. Totgeborene und Fehlgeborene werden anhand des Körpergewichts unterschieden, wobei erstere mind. $500 \mathrm{~g}$ schwer sind.

Tote Lebendgeborene und Totgeborene gelten rechtlich als Leichnam und bedürfen einer Leichenschau, einer entsprechenden Beurkundung und Bestattung - Fehlgeborene hingegen nicht.

\section{Veranlassung der Leichenschau}

Abhängig vom Ort des Todeseintritts ist die Leichenschau von Angehörigen, Mitbewohnern oder 
demjenigen, auf dessen Grundstück sich der Sterbefall ereignet hat, zu veranlassen. In Krankenhäusern, Heimen, Anstalten oder Verkehrsmitteln müssen die jeweiligen Leitungspersonen die Leichenschau in die Wege leiten.

\section{Wer darf bzw. muss die Leichenschau durchführen?}

Jeder Arzt ist prinzipiell verpflichtet | Auch hinsichtlich der Frage, wer eine Leichschau durchführen darf bzw. muss, gibt es Abweichungen zwischen den Bundesländern. Meist ist es von Vorteil, wenn der behandelnde Arzt des Verstorbenen die Leichenschau durchführt, da er die medizinische Vorgeschichte am besten kennt. Prinzipiell muss jeder Arzt auf Verlangen eine Leichenschau durchführen. Im Falle eines möglichen Behandlungsfehlervorwurfs ist es abhängig von der Regelung des Bundeslands, ob der behandelnde Arzt die Leichenschau ablehnen kann oder es ihm sogar verboten ist, sie durchzuführen.

Unvollständige Leichenschau | In einigen Bundesländern sind Notärzte im Dienst nur zu einer unvollständigen Leichenschau verpflichtet. Diese umfasst die Feststellung

- der Personalien,

- des Todes,

- der Todeszeit,

- des Todesortes und

- deren Dokumentation in einer ,vorläufigen Todesbescheinigung“ oder einer „Todesbescheinigung ohne Ursachenfeststellung“.

In solchen Fällen soll der Notarzt die Durchführung einer vollständigen Leichenschau durch einen anderen Arzt veranlassen.

\section{Dringlichkeit der Leichenschau}

Möglichst unverzüglich I Je nach Bundesland ist die Leichenschau unverzüglich oder innerhalb bestimmter Fristen auszuführen. Im juristischen Sprachgebrauch bedeutet unverzüglich „ohne schuldhaftes Zögern“. Das bedeutet, dass nur dringende, nicht aufschiebbare Maßnahmen noch erledigt werden dürfen. Eine gerade stattfindende Operation muss nicht zugunsten einer Leichenschau abgebrochen werden. Ein volles Wartezimmer rechtfertigt hingegen keinen Verzug. Sollte der beauftragte Arzt die Leichenschau zum Schutze eines höherwertigen Gutes (Pflichtenkollision) nicht oder nicht unverzüglich durchführen können, so muss er dafür sorgen, dass ein anderer Arzt die Leichenschau durchführt. Die in einigen Bundesländern festgelegten Fristen von 6,8 oder 12 Stunden, innerhalb derer die Leichenschau abgewickelt werden soll, werden angesichts der genannten Ziele der Leichenschau (insbesondere der Todesfeststellung) in der Literatur teilweise kritisch gesehen.

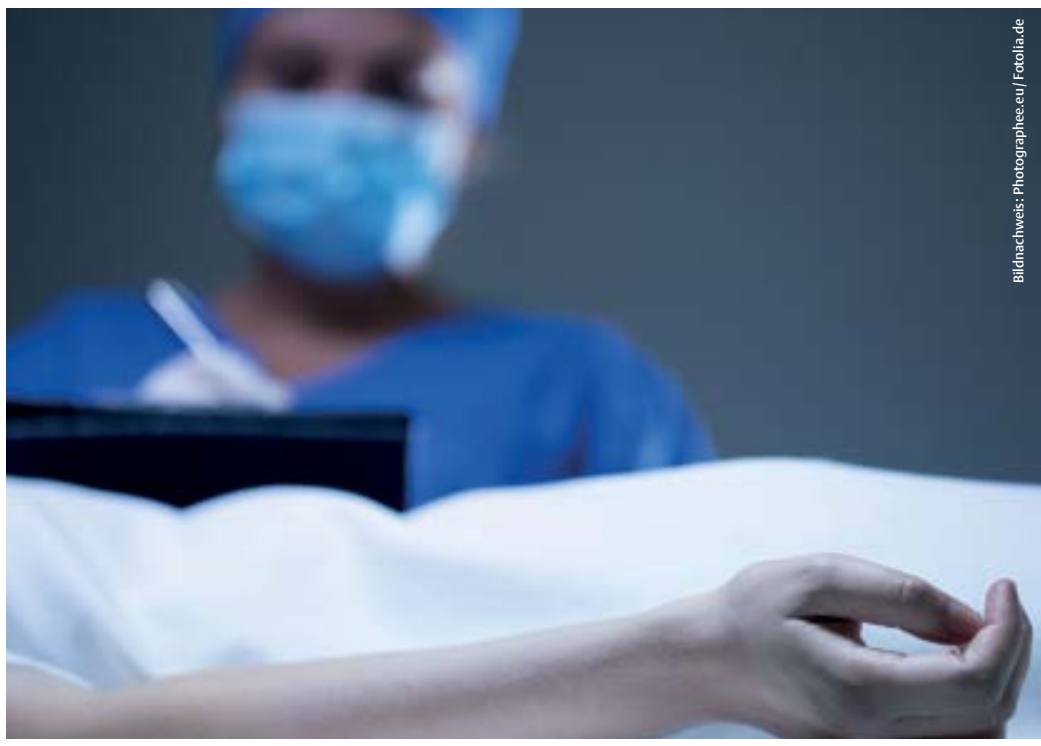

Die Leichenschau sollte möglichst am Leichenfundoder Sterbeort stattfinden. In

\section{Todesart und Todesursache}

Natürlicher und nicht-natürlicher Tod | Der Begriff Todesart trägt mehr der juristischen Denkweise Rechnung. Bei einem natürlichen Tod handelt es sich um einen Tod aus krankhafter, innerer Ursache, der unabhängig von rechtlich bedeutsamen Faktoren eingetreten ist. Ein nicht-natürlicher Tod liegt vor, wenn er auf äußere Einflüsse (Unfall, Suizid, Tötung) zurückgeführt werden kann - unabhängig davon, wie lange diese zurückliegen (z.B. Unfall $\bullet$ Krankenhaus $\$ Operation nach 4 Wochen verstorben).

Ungeklärte Todesart | Ist dem leichenschauenden Arzt diese Unterscheidung nicht möglich, so besteht in einigen Bundesländern die Möglichkeit, eine ungeklärte Todesart zu bescheinigen. Der leichenschauende Arzt muss seine Entscheidung rechtssicher bejahen können. Das heißt, seine Entscheidung muss für Außenstehende schlüssig nachvollziehbar sein.

Todesursache I Im Gegensatz zur Todesart ist die Todesursache ein medizinischer Begriff. Soweit möglich, soll aus einem Grundleiden die unmittelbare Todesursache nachvollziehbar und medizinisch schlüssig abgeleitet werden (z.B.: Alkoholmissbrauch $\bullet$ Leberzirrhose $\bullet$ Verbluten aus Ösophagusvarizen).

Es sollten möglichst keine unspezifischen Endzustände (Herzstillstand, Herz-KreislaufVersagen) genannt werden.

\section{Pflichten und Rechte des leichenschauenden Arztes}

Polizei benachrichtigen | Der leichenschauende Arzt hat bestimmte Meldepflichten zu beachten. Ausnahmefällen kann sie nach Feststellung des Todes zur weiteren Begutachtung an einen anderen Ort (z. B. in eine Leichenhalle) gebracht werden. 
Anna Isabelle Gutmann

ist wissenschaftliche Mitarbeiterin am Institut für Rechtsmedizin und Verkehrsmedizin an der Uniklinik Heidelberg.

Isabelle.Gutmann@med. uni-heidelberg.de

Dr. med.

Clara-Sophie Schwarz ist wissenschaftliche Mitarbeiterin am Institut für Rechtsmedizin und Verkehrsmedizin an der Uniklinik Heidelberg.

Prof. Dr. med. univ. Kathrin Yen ist Ärztliche Direktorin am Institut für Rechtsmedizin und Verkehrsmedizin an der Uniklinik Heidelberg.

Dr. med. Roman Bux, MBA ist Oberarzt am Institut für Rechtsmedizin und Verkehrsmedizin an der Uniklinik Heidelberg.
Dr. med.

Stellt er einen nicht-natürlichen oder ungeklärten Tod fest, so muss er die Leichenschau abbrechen und die Polizei informieren, damit das Spurenbild am Leichenfundort nicht weiter verändert wird. Auch bei unbekannter Identität des Leichnams muss er die Polizei benachrichtigen.

Infektionen und Berufserkrankung I Das Infektionsschutzgesetz sieht für eine Reihe von Erkrankungen eine namentliche oder nicht-namentliche Meldung vor, die binnen 24 Stunden an das Gesundheitsamt zu erfolgen hat. Weiterhin ist bereits der Verdacht, dass eine Person an den Folgen oder direkt an einer Berufserkrankung verstorben ist, der zuständigen Berufsgenossenschaft zu melden ( $§ 202$ SGB VII).

Ort der Leichenschau | Grundsätzlich sollte die Leichenschau am Leichenfund- oder Sterbeort stattfinden. Durch den Einbezug der Umgebung wird eine bestmögliche Beurteilung der Befunde gewährleistet. Aus diesem Grund wird dem leichenschauenden Arzt ein Betretungsrecht zugestanden, das im Bedarfsfall mit polizeilicher Hilfe auch gegen Widerstand durchgesetzt werden kann. Ist der Ort für eine Leichenschau ungeeignet (z.B. bei großem Publikumsverkehr), so darf der Leichnam nach Feststellung des Todes zur weiteren Durchführung der Leichenschau an einen besser geeigneten Ort (z.B. Leichenhalle) gebracht werden.

Auskunftsrecht | Daneben hat der leichenschauende Arzt Anspruch auf Auskünfte (Auskunftsrecht) von Angehörigen oder behandelnden Ärzten bzw. medizinischem Personal. Insbesondere, wenn ihm der Verstorbene nicht persönlich bekannt gewesen ist, ist er auf diese Informationen zum vollständigen Ausfüllen der Todesbescheinigung angewiesen. Zu diesem Zweck darf die ärztliche Schweigepflicht von ehemals behandelnden Ärzten gebrochen werden.

\section{Todesbescheinigung und Stellenwert der Todeszeit}

Die bei der Leichenschau festgestellten Befunde sind in der Todesbescheinigung zu dokumentieren. Diese gliedert sich in einen nicht-vertraulichen Teil mit Angaben zu den Personalien des Verstorbenen, Todesart und Todeszeit, und einen vertraulichen Teil, in welchem unter anderem die Todesursache mitgeteilt wird.

Der nicht-vertrauliche Teil dient als Grundlage zur Beurkundung des Todesfalles im Standesamt; der vertrauliche Teil ist unter anderem für das Gesundheitsamt bestimmt.

DOI 10.1055/s-0041-102207 Dtsch Med Wochenschr 2015; 140: 852-854

(C) Georg Thieme Verlag KG . Stuttgart · New York · ISSN 0012-0472 so weit wie möglich einzugrenzen (z. B. durch Angabe des letzten Kontakts und des Auffindezeitpunkts). Die Angabe der Todeszeit hat besondere Bedeutung bei der Überprüfung von Alibis im Rahmen von Ermittlungen oder bei der Festlegung einer Erbfolge. Eine Todeszeitschätzung anhand der Leichenerscheinungen sollte in speziellen Fällen hingegen einem Rechtsmediziner vorbehalten bleiben bzw. mit der gebotenen Zurückhaltung erfolgen. Bei einer erfolglosen Reanimation ist als Todeszeit der Zeitpunkt anzugeben, an dem die Maßnahme beendet wurde.

\section{Welche Konsequenzen drohen?}

Geld- und Freiheitsstrafen | Bei nicht lege artis durchgeführter Leichenschau kann es zu empfindlichen Strafen kommen. Ordnungswidrigkeiten in Form von nicht rechtzeitig oder unzureichend durchgeführten Leichenschauen oder die Missachtung von Meldepflichten können mit Geldstrafen von 5-25000€ sanktioniert werden. Weiterhin kann ein vorsätzliches oder fahrlässiges falsches Ausfüllen der Todesbescheinigung den Tatbestand einer Falschbeurkundung mit einer Freiheitsstrafe bis zu einem Jahr ( $\$ 271$ StGB) erfüllen.

Fahrlässige Tötung | Wenn weitere Personen versterben - etwa wenn eine Kohlenmonoxidquelle oder eine gefährliche Stromquelle nicht entdeckt wird - und dies durch eine sorgfältig durchgeführte Leichenschau hätte vermieden werden können, so muss mit einer Freiheitsstrafe von bis zu fünf Jahren wegen fahrlässiger Tötung ( $\$ 222$ StGB) gerechnet werden.

Fazit

- Die Leichenschau hat weitreichende Konsequenzen und muss daher sorgfältig und verantwortungsbewusst durchgeführt werden.

- Jedes Bundesland regelt die gesetzlichen Bestimmungen der Leichenschau selbst.

- Tote Lebendgeborene und Totgeborene gelten rechtlich als Leichnam und bedürfen einer Leichenschau - Fehlgeborene nicht.

- Prinzipiell muss jeder Arzt auf Verlangen eine Leichenschau durchführen.

- Bei nicht-natürlichem oder ungeklärtem Tod oder wenn die Identität des Leichnams unbekannt ist, muss die Polizei benachrichtigt werden.

- Der leichenschauende Arzt hat ein Betretungsund Auskunftsrecht.

- Bei nicht lege artis durchgeführter Leichenschau kann es zu empfindlichen Strafen kommen. 